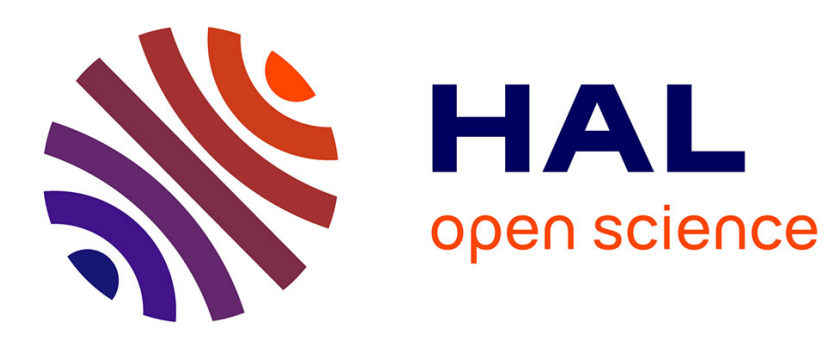

\title{
A note on the characterization of optimal allocations in OLG models with multiple goods
}

\author{
Jean-Marc Bonnisseau, Lalaina Rakotonindrainy
}

\section{To cite this version:}

Jean-Marc Bonnisseau, Lalaina Rakotonindrainy. A note on the characterization of optimal allocations in OLG models with multiple goods. 2015. halshs-01158117v2

\section{HAL Id: halshs-01158117 \\ https://shs.hal.science/halshs-01158117v2}

Submitted on 29 Oct 2015

HAL is a multi-disciplinary open access archive for the deposit and dissemination of scientific research documents, whether they are published or not. The documents may come from teaching and research institutions in France or abroad, or from public or private research centers.
L'archive ouverte pluridisciplinaire HAL, est destinée au dépôt et à la diffusion de documents scientifiques de niveau recherche, publiés ou non, émanant des établissements d'enseignement et de recherche français ou étrangers, des laboratoires publics ou privés. 


\section{A \\ UNIVERSITÉ PARIS 1}

PANTHÉON SORBONNE
Documents de Travail du Centre d'Economie de la Sorbonne

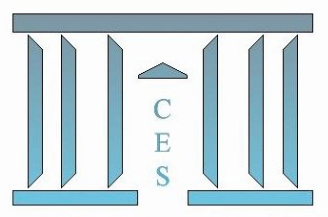

A note on the characterization of optimal allocations in OLG models with multiple goods

Jean-Marc BoNNISSEAU, Lalaina RAKOTONINDRAINY

\author{
2015.03R
}

Version révisée

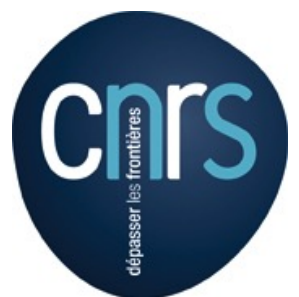




\title{
A note on the characterization of optimal allocations in OLG models with multiple goods *
}

\author{
Jean-Marc Bonnisseau and Lalaina Rakotonindrainy ${ }^{\dagger}$
}

July 2015

\begin{abstract}
We consider a pure exchange overlapping generations economy with a varying number of commodities and consumers per period having possibly non-complete non transitive preferences. We provide a geometric and direct proof of the Balasko-Shell characterization of Pareto optimal allocation. As a by-product, we compute an explicit Pareto improving transfer when the criterion is not satisfied, which is minimal for some suitable distance.

JEL classification: C62, D50, D62.

Keywords: Overlapping generations model, non complete non transitive preferences, normal cone, equilibrium, Pareto optimality.
\end{abstract}

\section{Introduction}

As already well known, OLG equilibrium allocations may lack in being Pareto optimal. This market failure was established by Samuelson [23], who attributes this phenomenon as a lack of double coincidence of wants, and suggests to solve this by using the role of money. Geanakoplos [16] clarifies this phenomenon as a result of two facts: generations overlap and infinite horizon. He points some links between the Samuelson model and the Arrow-Debreu model, and

\footnotetext{
${ }^{*}$ This paper is a modest contribution for this special issue in honor of Lionel Thibault, whose tremendous works in non-smooth analysis have a long lasting influence in the developments of mathematical economics since many years. This work was supported by the French National Research Agency, through the program Investissements d'Avenir, ANR-10-LABX-93-01. We would like to thank Mich Tvede, Alfred Greiner and anonymous referees for useful comments and suggestions.

${ }^{\dagger}$ Paris School of Economics, Université Paris 1 Panthéon-Sorbonne, Centre d'Economie de la Sorbonne-CNRS, 106-112 Boulevard de l'Hôpital, 75647 Paris Cedex 13, France. E-mail: Jean-Marc.Bonnisseau@univ-paris1.fr, Lalaina.Rakotonindrainy@malix.univ-paris1.fr
} 
established how a durable good such as money, or an infinitely lived asset like a land, could restore the market failure.

Balasko and Shell [3] provide a criterion based on the asymptotic behavior of the norm of the prices to characterize Pareto optimal allocation without durable good or infinitely lived asset. They assume that the preferences are represented by utility functions so are transitive and complete. Burke [10] revisits this criterion by focusing in particular on the right definition of the Gaussian curvature of the indifference surface. Actually, these authors provide a proof with a first step considering the special case of a single commodity per period. Then, the generalization to several commodities is only sketched.

Our purpose in this paper is to consider a varying number of commodities per period, several consumers for each generation and non-complete, nontransitive preferences, taking advantages of a simpler direct and more geometric proof of the Balasko- Shell Criterion. Furthermore, as a by-product of the proof, we compute explicitly a Pareto improving transfer when the allocation does not satisfy the Balasko-Shell Criterion. Nevertheless, note that the structure of the proof is strongly based on Balasko-Shell's one.

Allowing for preferences to be non-complete and non-transitive is interesting since it is less demanding with respect to the rationality axiom. Indeed, many examples have been given showing that transitivity or completeness of preferences may not hold. In our case where agents are facing multi attribute comparisons for multiple goods, their preferences may contain cycles, thus lack in being transitive, and they may not be able to compare bundles between them, since an agent may strictly prefer bundles with more of all goods but may not be able to rank bundles where there is a trade off between these goods.

More precisely, non-complete preferences have been considered in the context of choice under uncertainty. Indeed, an agent may not be able to have a precise guess about the state of the world, leading her to be unable to compare two objects. The incompleteness is then related to "indecisiveness in beliefs", which is what Bewley [4] tried to capture in his model of uncertainty. Another type of non-complete preference, called "indecisiveness in tastes" is described by Aumann [1], in which case it is due to an inability to compare some certain outcomes. More recently, Mandler [17, 18] pointed out the need to distinguish types of preferences for a better understanding of why preferences are noncomplete. Indeed, if the preference of an agent is based on her choice, that is $x$ is preferred to $y$ if $x$ is chosen over $y$ then it is necessarily complete, but if it is based on her knowledge, meaning that $x$ is known to be better than $y$, then the preference needs not be complete since the agent may not have such a knowledge. Examples of applications of non-complete preferences can be seen in many works of Bewley, for instance, they can be applied to dynamic choices [5], to econometric inference [6] and to innovation and entrepreneurship [7]. 
Whenever the axioms of transitivity or completeness are removed, the utility function does not exist, so that the demand cannot be characterized as the solution of an optimization problem. It is now a maximal element of a set-valued mapping and a fixed-point like theorem is required to prove its non-emptiness. Andreu Mas-Colell (1974) is one of the pioneers who tried to get rid of these two axioms for the existence of competitive equilibrium. To do so, with David Gale (see [14]), they have to use a maximal element theorem for lower semicontinuous set-valued mappings instead of the the standard Kakutani's fixed point theorem.

To study optimality conditions, the absence of transitivity and completeness is less problematic since we are working with a reference allocation and so, given preferred sets. We do not have to deal with the behavior of the demand with respect to price change. Nevertheless, the lack of representation by a utility function prevent us to use the standard differentiability assumption and the link between the curvature of the indifference surfaces and the second derivative of utility function. So we adopt a geometric approach to state the assumptions directly on the preferred sets. The smoothness assumption is obtained by assuming that the normal cone is an half line. For the upper bound of the curvature, we use the notion of prox-regularity of the complementary of the preferred set, introduced in variational analysis by Rockafellar and Poliquin in [20], which is extensively studied by Thibault and Colombo in [11]. For the lower bound, which is related to the strict convexity of the preferred sets, we assume that the truncated preferred sets are included in a suitable ball with a large enough radius.

Note that an approach based on preferred sets has already been provided by Borglin and Keiding [9] and by Borglin [8]. Indeed, even if initially they present the model with utility functions, they introduce the notion of "reduced models", that actually consist of the "no-worse-than sets". They consider characterizations based on parameters that describe the economy such as curvatures measures, and the efficiency of the reduced models are simplified by considering an inner and outer approximation of the no-worse-than sets. In the case where the approximation consists of hyperbolas, they obtain parametric efficiency criteria, that is, based on the parameters of the reduced models. So, this work can be easily extended to non complete non transitive preferences.

Our main contribution is the simplification of the original proof of Balasko and Shell and the fact that we directly tackle the multi-commodity case. It allows us to consider a varying number of commodities at each period. Actually we show that the parameter $\alpha^{t}$ used by Balasko and Shell to describe the Pareto improving transfer has a nice geometric interpretation in terms of a radius of a sphere tangent to the boundary of the preferred set at the allocation. This remark leads us to use this radius as a particular distance function and to 
compute explicitly the minimal Pareto improving transfer when the criterion is not satisfied.

Details about the model and assumptions are described in Section 2. Some preliminary results are provided in Section 3. In particular, we characterize the weak Pareto optimal allocation in term of the existence of a supporting price using the normal cone to the preferred sets. The end of this Section explains how the multi-consumer case can be simplified by considering aggregate feasible Pareto improving transfers. In Section 4, we provide the proof and we show which assumptions are used for the if part and for the only if part of the criterion. We also provide two examples showing that the result is no more true if one of the conditions on the curvature does not hold true.

We consider this contribution as a first step to be able in future works to tackle the question in presence of durable commodities and with heterogeneous longevities of the agents.

\section{The model}

The following notations are used throughout the paper. We consider several finite dimensional Euclidean space $\mathbb{R}^{L}$. In each of them, $\mathbb{R}_{+}^{L}$ is the standard positive cone and $\mathbb{R}_{++}^{L}$ its interior. $x \leq y$ means that $y-x \in \mathbb{R}_{+}^{L}, x \cdot y$ denotes the standard inner product of the vectors $x$ and $y,\|x\|=\sqrt{x \cdot x}$ denotes the standard Euclidean norm. If $P$ is a subset of $\mathbb{R}^{L}, \bar{P}$ denotes its closure and $\operatorname{int} P$, its interior. $B(x, r)$ (resp. $\bar{B}(x, r))$ denotes the open (resp. closed) ball of center $x$ and radius $r$.

We consider an OLG economy $\mathcal{E}$ with infinitely many dates $t=1,2 \ldots$ At each date there is a finite set of commodities $\mathcal{L}_{t}$, and we denote by $L_{t}$ its cardinal.

At each date $t \in \mathbb{N}$, a nonempty finite set of individuals $\mathcal{I}_{t}$ is born, living for two periods, young at date $t$ and old at date $t+1$. We start with the first generation 0 who lives for only one period and consists of the old agents at date 1. $\mathcal{I}=\cup_{t=0}^{\infty} \mathcal{I}_{t}$ denotes the set of all individuals and $\mathcal{I}_{-0}=\cup_{t=1}^{\infty} \mathcal{I}_{t}$.

At each date $t \geq 1$, we denote by $x^{i}=\left(x_{t}^{i}, x_{t+1}^{i}\right)$ the consumption by an individual $i$ in $\mathcal{I}_{t}$, which is an element of the consumption set $X^{i}=\mathbb{R}_{+}^{L_{t}} \times \mathbb{R}_{+}^{L_{t+1}}$. The consumption set of consumers of generation 0 is $X^{i}=\mathbb{R}_{+}^{L_{1}}$.

Consumers preferences are represented by a (strict) preference relation $P^{i}$ : $X^{i} \rightarrow X^{i}$. For all $i \in \mathcal{I}$ :

$\xi^{i} \in P^{i}\left(x^{i}\right)$ means that $\xi^{i}$ is strictly preferred to $x^{i}$.

$\xi^{i} \in \bar{P}^{i}\left(x^{i}\right)$, the closure of $P^{i}\left(x^{i}\right)$, means that $\xi^{i}$ is preferred or indifferent to $x^{i}$.

For all $i \in \mathcal{I}$, we denote by $N_{\bar{P}^{i}\left(x^{i}\right)}\left(x^{i}\right)$ the normal cone of $\bar{P}^{i}\left(x^{i}\right)$ at $x^{i}$, that 
is,

$$
N_{\bar{P}^{i}\left(x^{i}\right)}\left(x^{i}\right)=\left\{q \in \mathbb{R}^{L_{t}} \times \mathbb{R}^{L_{t+1}} \mid q \cdot\left(z-x^{i}\right) \leq 0, \forall z \in \bar{P}^{i}\left(x^{i}\right)\right\}
$$

We now posit the main assumption, which is maintained throughout the paper.

\section{Assumption A.}

a) For all individual $i$ in $\mathcal{I}, P^{i}\left(x^{i}\right)$ is open in $X^{i}$, convex, $x^{i} \in \bar{P}^{i}\left(x^{i}\right) \backslash P^{i}\left(x^{i}\right)$ and $P^{i}\left(x^{i}\right)+X^{i} \subset P^{i}\left(x^{i}\right)$.

b) Each consumer $i$ has some endowments $e^{i}$ of the goods during his lifetime which belongs to her consumption set $X^{i}$. For each period $t \geq 1, e_{t}$ denotes the total endowments at this date, that is, $e_{t}=\sum_{i \in \mathcal{I}_{t-1} \cup \mathcal{I}_{t}} e_{t}^{i}$.

c) For all $i \in \mathcal{I}$, for all $x^{i}$ in the interior of $X^{i},-N_{\bar{P}^{i}\left(x^{i}\right)}\left(x^{i}\right)$ is a half line $\left\{t \gamma^{i}\left(x^{i}\right) \mid t \geq 0\right\}$, defined by $\gamma^{i}\left(x^{i}\right)$ which is a continuous mapping on the interior of $X^{i}$ satisfying $\left\|\gamma^{i}\left(x^{i}\right)\right\|=1$. For all $x^{i}$ in the interior of $X^{i}$, for all $i \in \mathcal{I}_{-0},, \gamma^{i}\left(x^{i}\right) \in \mathbb{R}_{++}^{L_{t}} \times \mathbb{R}_{++}^{L_{t+1}}$ and for all $i \in \mathcal{I}_{0}, \gamma^{i}\left(x^{i}\right) \in \mathbb{R}_{++}^{L_{1}}$.

Assumption A is a classical assumption in a standard finite economy. If the preferences are represented by a utility function, it means that it is continuous, quasi-concave, strictly increasing and smooth on the interior of $X^{i}$.

At each date $t$, there is a spot market for the $L_{t}$ commodities. The spot price vector $p$ is an element of $\prod_{t=1}^{\infty} \mathbb{R}_{++}^{L_{t}}$ and $p_{t \ell}$ is the spot price of commodity $\ell$ at date $t$. We consider the set of normalized prices $\Delta:=\left\{p \in \prod_{t=1}^{\infty} \mathbb{R}_{++}^{L_{t}} \mid\right.$ $\left.\left\|p_{1}\right\|=1\right\}$. We denote by $\Pi_{t}=\left(p_{t}, p_{t+1}\right)$, for $t \geq 1$, and $\Pi_{0}=p_{1}$.

\section{Budget Constraints}

The budget constraint at a given price $p$, for each agent $i \in \mathcal{I}_{-0}$ is given by:

$$
\Pi_{t} \cdot x^{i}=p_{t} \cdot x_{t}^{i}+p_{t+1} \cdot x_{t+1}^{i} \leq \Pi_{t} \cdot e^{i}=p_{t} \cdot e_{t}^{i}+p_{t+1} \cdot e_{t+1}^{i},
$$

and for each agent $i \in \mathcal{I}_{0}$,

$$
\Pi_{0} \cdot x^{i}=p_{1} \cdot x_{1}^{i} \leq \Pi_{0} \cdot e^{i}=p_{1} \cdot e_{1}^{i} .
$$

\section{Preliminary results}

Let us recall some basic definitions of equilibrium and optimal allocations in a standard pure exchange OLG economy with multiple commodities. An allocation $\left(x^{i}\right) \in \prod_{t \geq 1} \prod_{i \in \mathcal{I}_{t}} X^{i}$ is feasible if $\sum_{i \in \mathcal{I}_{t-1} \cup \mathcal{I}_{t}} x_{t}^{i *}=e_{t}=\sum_{i \in \mathcal{I}_{t-1} \cup \mathcal{I}_{t}} e_{t}^{i}$ for all $t \geq 1$ and it is interior if $x^{i} \in \operatorname{int} X^{i}$ for all $i \in \mathcal{I}$. 
Definition 1 An equilibrium of the economy $\mathcal{E}$ is a list $\left(p^{*},\left(x^{i *}\right)\right)$ in $\Delta \times$ $\prod_{t=0}^{\infty} \prod_{i \in \mathcal{I}_{t}} X^{i}$ such that:

a) for all $i \in \mathcal{I},\left(x^{i *}\right)$ is a maximal element for $P^{i}$ in the budget set associated to the equilibrium price $p^{*}$, that is, $\Pi_{t} \cdot x^{i *} \leq \Pi_{t} \cdot e^{i}$, and for all $x^{i} \in P^{i}\left(x^{i *}\right)$, $\Pi_{t} \cdot x^{i}>\Pi_{t} \cdot e^{i}$.

b) the allocation $\left(x^{i *}\right)$ is feasible:

$$
\sum_{i \in \mathcal{I}_{t-1} \cup \mathcal{I}_{t}} x_{t}^{i *}=e_{t} \text { for } t \geq 1
$$

Proposition 1 Under Assumption A, if the initial endowments are strictly positive, the $O L G$ economy $\mathcal{E}$ has an equilibrium.

The existence of an equilibrium can be proved following the same procedure as in [2]. The existence of an equilibrium in the truncated economies is obtained by using the existence result in finite dimension of Gale and Mas-Colell [14], [15], see also Florenzano [13]. Then, the limit argument follows the same steps using the fact that the prices and the allocations of the equilibrium in the truncated economies remain in compact sets for the product topology.

Definition 2 The feasible allocation $x$ in $\prod_{i \in \mathcal{I}} X^{i}$ is Pareto optimal (PO) (resp. weakly Pareto optimal (WPO)) if there is no $\left(y^{i}\right)$ in $\prod_{i \in \mathcal{I}} X^{i}$ such that:

$$
\sum_{i \in \mathcal{I}_{t-1} \cup \mathcal{I}_{t}} y_{t}^{i}=e_{t}, \text { for } t \geq 1
$$

and for all $i \in \mathcal{I}, y^{i} \in \bar{P}^{i}\left(x^{i}\right)$, with $y^{i} \in P^{i}\left(x^{i}\right)$ for at least one individual $i$ (resp. and there exists $\underline{t} \geq 1$ such that for all $t \geq \underline{t}$, for all $i \in \mathcal{I}_{t}, y^{i}=x^{i}$ ).

We remark that a PO allocation is WPO.

Definition 3 Let $x=\left(x^{i}\right)$ be an allocation in $\prod_{i \in \mathcal{I}} X^{i}$. The price $p \in \Delta$ is said to support $x$ if for each $t \in \mathbb{N}$, for all $i \in \mathcal{I}_{t}$ and for all $\xi^{i} \in P^{i}\left(x^{i}\right)$, $\Pi_{t} \cdot \xi^{i}>\Pi_{t} \cdot x^{i}$.

Remark 1 Every competitive allocation $x^{*}=\left(x^{i *}\right)$ associated with the equilibrium price $p^{*} \in \Delta$ is supported by $p^{*}$.

If $x=\left(x^{i}\right)$, an interior allocation, is supported by the price $p$, then for all $i \in \mathcal{I}$, there exists $\lambda^{i}>0$ such that $\gamma^{i}\left(x^{i}\right)=\lambda^{i} \Pi_{t}$.

Lemma 1 If $x=\left(x^{i}\right)$, an interior allocation, is WPO, then, for all $t \geq 0$, $\gamma^{i}\left(x^{i}\right)=\gamma^{j}\left(x^{j}\right)$ for all $i, j \in \mathcal{I}_{t}$.

Proof. Let $t \geq 0$. Since $\left(x^{i}\right)$ is WPO, $\sum_{i \in \mathcal{I}_{t}} x^{i} \notin \sum_{i \in \mathcal{I}_{t}} P^{i}\left(x^{i}\right)$. Indeed, if it would not hold, then there exists $\left(\xi^{i}\right)_{i \in \mathcal{I}_{t}} \in \prod_{i \in \mathcal{I}_{t}} P^{i}\left(x^{i}\right)$, such that $\sum_{i \in \mathcal{I}_{t}} x^{i}=$ 
$\sum_{i \in \mathcal{I}_{t}} \xi^{i}$. Then, one easily checks that the allocation $\left(y^{i}\right)$ defined by $y^{i}=x^{i}$ for $i \notin \mathcal{I}_{t}$ and $y^{i}=\xi^{i}$ for $i \in \mathcal{I}_{t}$ is feasible and Pareto dominates $\left(x^{i}\right)$, which is in contradiction with the weak Pareto optimality of $\left(x^{i}\right)$.

By Assumption A, $\left(P^{i}\left(x^{i}\right)\right)_{i \in \mathcal{I}_{t}}$ are convex and nonempty, and for all $i$, $x^{i} \notin P^{i}\left(x^{i}\right)$ and $x^{i} \in \bar{P}^{i}\left(x^{i}\right)$. So, for each $i \in \mathcal{I}_{t}$, there exists a sequence $\left(\xi^{i \nu}\right)$ of $P^{i}\left(x^{i}\right)$, which converges to $x^{i}$. The set $\sum_{i \in \mathcal{I}_{t}} P^{i}\left(x^{i}\right)$ being convex, so by using the standard separation theorem for convex sets in finite-dimensional space for $\sum_{i \in \mathcal{I}_{t}} x^{i}$ and $\sum_{i \in \mathcal{I}_{t}} P^{i}\left(x^{i}\right)$, there exists $q \neq 0$ such that $q \cdot \sum_{i \in \mathcal{I}_{t}} x^{i} \leq$ $q \cdot \sum_{i \in \mathcal{I}_{t}} \xi^{i}$ for all $\left(\xi^{i}\right) \in \prod_{i \in \mathcal{I}_{t}} P^{i}\left(x^{i}\right)$. Consider an individual $i_{0}$ in $\mathcal{I}_{t}$. Then for all $\xi^{i_{0}} \in P^{i_{0}}\left(x^{i_{0}}\right), q \cdot x^{i_{0}}+q \cdot \sum_{i \in \mathcal{I}_{t}, i \neq i_{0}} x^{i} \leq q \cdot \xi^{i_{0}}+q \cdot \sum_{i \in \mathcal{I}_{t}, i \neq i_{0}} \xi^{i \nu}$. By taking the limit, we obtain: $q \cdot x^{i_{0}} \leq q \cdot \xi^{i_{0}}$, which means that $q$ belongs to the cone $-N_{\bar{P}^{i_{0}}\left(x^{\left.i_{0}\right)}\right.}\left(x^{i_{0}}\right)$, thus $q=\|q\| \gamma^{i_{0}}\left(x^{i_{0}}\right)$. By repeating the same reasoning for all individuals of $\mathcal{I}_{t}$, we establish that $\gamma^{i}\left(x^{i}\right)=\frac{1}{\|q\|} q$ for all $i \in \mathcal{I}_{t}$.

Lemma 2 The interior allocation $x=\left(x^{i}\right)$ is WPO if and only if there exists a price sequence $p \in \Delta$ which supports $x=\left(x^{i}\right)$.

Proof. Let $x$ be supported by a price sequence $p$. Assume that $x$ is not WPO, then there exists a feasible allocation $\left(y^{i}\right)$ and some $\underline{t} \geq 1$ such that $x^{i}=y^{i}$ for all $i \in \mathcal{I}_{t}, t \geq \underline{t}$, where $y^{i} \in \bar{P}^{i}\left(x^{i}\right)$ for all $i \in \mathcal{I}$ and $y^{i} \in P^{i}\left(x^{i}\right)$ for at least an individual. Therefore, for all $t$ and for all $i \in \mathcal{I}_{t}, \Pi_{t} \cdot y^{i} \geq \Pi_{t} \cdot x^{i}$ with at least one strict inequality for $i_{0} \in \mathcal{I}_{t_{0}}$ where $t_{0}<\underline{t}$. Thus, we have:

$$
\begin{gathered}
p_{1} \cdot \sum_{i \in \mathcal{I}_{0} \cup \mathcal{I}_{1}} y_{1}^{i}+p_{2} \cdot \sum_{i \in \mathcal{I}_{1} \cup \mathcal{I}_{2}} y_{2}^{i}+\ldots+p_{\underline{t}-1} \cdot \sum_{i \in \mathcal{I}_{\underline{t}-1} \cup \mathcal{I}_{\underline{\underline{t}}-2}} y_{\underline{\underline{t}}-1}^{i}+p_{\underline{\underline{t}}} \cdot \sum_{i \in \mathcal{I}_{\underline{t}-1}} y_{\underline{\underline{t}}}^{i}> \\
p_{1} \cdot \sum_{i \in \mathcal{I}_{0} \cup \mathcal{I}_{1}} x_{1}^{i}+p_{2} \cdot \sum_{i \in \mathcal{I}_{1} \cup \mathcal{I}_{2}} x_{2}^{i}+\ldots+p_{\underline{t}-1} \cdot \sum_{i \in \mathcal{I}_{\underline{\underline{t}}-1} \cup \mathcal{I}_{\underline{\underline{t}}-2}} x_{\underline{t}-1}^{i}+p_{\underline{t}} \cdot \sum_{i \in \mathcal{I}_{\underline{t}-1}} x_{\underline{t}}^{i}
\end{gathered}
$$

Since, $y_{t}^{i}=x_{t}^{i}$ for all $i \in \mathcal{I}_{t}, t \geq \underline{t}$, we get:

$$
\begin{aligned}
& p_{1} \cdot \sum_{i \in \mathcal{I}_{0} \cup \mathcal{I}_{1}} y_{1}^{i}+p_{2} \cdot \sum_{i \in \mathcal{I}_{1} \cup \mathcal{I}_{2}} y_{2}^{i}+\ldots+p_{\underline{t}-1} \cdot \sum_{i \in \mathcal{I}_{\underline{\underline{t}}-1} \cup \mathcal{I}_{\underline{t}-2}} y_{\underline{t}-1}^{i}+p_{\underline{\underline{t}}} \cdot \sum_{i \in \mathcal{I}_{\underline{t}-1} \cup \mathcal{I}_{\underline{t}}} y_{\underline{\underline{t}}}^{i}> \\
& p_{1} \cdot \sum_{i \in \mathcal{I}_{0} \cup \mathcal{I}_{1}} x_{1}^{i}+p_{2} \cdot \sum_{i \in \mathcal{I}_{1} \cup \mathcal{I}_{2}} x_{2}^{i}+\ldots+p_{\underline{t}-1} \cdot \sum_{i \in \mathcal{I}_{\underline{t}-1} \cup \mathcal{I}_{\underline{\underline{t}}-2}} x_{\underline{t}-1}^{i}+p_{\underline{t}} \cdot \sum_{i \in \mathcal{I}_{\underline{\underline{t}}-1} \cup \mathcal{I}_{\underline{\underline{t}}}} x_{\underline{t}}^{i}
\end{aligned}
$$

which is in contradiction with the feasibility of $\left(x^{i}\right)$ and $\left(y^{i}\right)$ implying

$$
\sum_{i \in \mathcal{I}_{t-1} \cup \mathcal{I}_{t}} x_{t}^{i}=\sum_{i \in \mathcal{I}_{t-1} \cup \mathcal{I}_{t}} y_{t}^{i}=e_{t}
$$

Conversely, let $x$ be a WPO allocation. We first truncate the economy at a finite horizon $t$ by considering the $t$ first generations. Denote $\mathcal{J}_{t-1}=\prod_{\tau=0}^{t-1} \mathcal{I}_{\tau}$. We shall prove the result by induction on the truncation at $t$.

First, consider the truncated economy $\mathcal{E}_{1}$ at date $t=1$, which consists of the generation $0, \mathcal{I}_{0}$. From Lemma $1, p_{1}=\gamma^{i}\left(x^{i}\right)$ for any $i \in \mathcal{I}_{0}$ supports $\left(x^{i}\right)_{i \in \mathcal{I}_{0}}$. 
Now, suppose that $\left(p_{1}, \ldots p_{t}\right)$ is supporting $\left(x^{i}\right)_{i \in \mathcal{J}_{t-1}}$, and let us prove that there is a unique $p_{t+1} \gg 0$ such that $\left(p_{1}, \ldots p_{t+1}\right)$ supports $\left(x^{i}\right)_{i \in \mathcal{J}_{t}}$. From Lemma 1 , for any $i_{0} \in \mathcal{I}_{t}, \gamma^{i_{0}}\left(x^{i_{0}}\right)$ supports $x^{i}$ for all $i \in \mathcal{I}_{t}$. So, it suffices to prove that $\gamma_{t}^{i_{0}}\left(x^{i_{0}}\right)$ is collinear to $p_{t}$ and then to choose $p_{t+1}=$ $\frac{\left\|p_{t}\right\|}{\left\|\gamma_{t}^{i_{0}}\left(x^{i_{0}}\right)\right\|} \gamma_{t+1}^{i_{0}}\left(x^{i_{0}}\right)$.

We consider a reduced economy with $L_{t}$ commodities, the individuals in $\mathcal{I}_{t-1} \cup \mathcal{I}_{t}$ and the preferences defined by $Q^{i}\left(\xi^{i}\right)=P^{i}\left(x_{t-1}^{i}, \xi^{i}\right)$ for $i \in \mathcal{I}_{t-1}$ and $Q^{i}\left(\xi^{i}\right)=P^{i}\left(\xi^{i}, x_{t+1}^{i}\right)$ for $i \in \mathcal{I}_{t}$. Since $x$ is a WPO allocation, the allocation $\left(x_{t}^{i}\right)_{i \in \mathcal{I}_{t-1} \cup \mathcal{I}_{t}}$ is Pareto optimal in this finite economy. We also remark that $-N_{Q^{i}\left(x_{t}^{i}\right)}\left(x_{t}^{i}\right)=\left\{\lambda \gamma_{t}^{i}\left(x_{t-1}^{i}, x_{t}^{i}\right) \mid \lambda \geq 0\right\}$ for $i \in \mathcal{I}_{t-1}$ and $-N_{Q^{i}\left(x_{t}^{i}\right)}\left(x_{t}^{i}\right)=$ $\left\{\lambda \gamma_{t}^{i}\left(x_{t}^{i}, x_{t+1}^{i}\right) \mid \lambda \geq 0\right\}$ for $i \in \mathcal{I}_{t}$. So, using the same argument as in the proof of Lemma 1, we prove that the vectors $\left(\left(\gamma_{t}^{i}\left(x_{t-1}^{i}, x_{t}^{i}\right)\right)_{i \in \mathcal{I}_{t-1}}\right.$ and $\left(\gamma_{t}^{i}\left(x_{t}^{i}, x_{t+1}^{i}\right)\right)_{i \in \mathcal{I}_{t}}$ are colinear. Since $\left(p_{t-1}, p_{t}\right)$ supports $x^{i}$ for any $i \in \mathcal{I}_{t-1}, p_{t}$ is colinear to $\gamma_{t}^{i}\left(x_{t-1}^{i}, x_{t}^{i}\right)$, so it is also colinear to $\gamma_{t}^{i}\left(x_{t}^{i}, x_{t+1}^{i}\right)$ for all $i \in \mathcal{I}_{t}$.

\section{Definition of aggregate Pareto improving transfers}

The proof of the main result studies the behavior of Pareto improving transfers that we now introduce. In the following, we distinguish transfers and aggregate transfers. For an allocation $\left(x^{i}\right)$, for each generation $t$, we define an aggregate preferred set as follows:

$$
\bar{P}_{t}\left(\left(x^{i}\right)\right):=\sum_{i \in \mathcal{I}_{t}} \bar{P}^{i}\left(x^{i}\right)
$$

From Assumption A, $\bar{P}_{t}\left(\left(x^{i}\right)\right)$ is a closed convex subset of $\mathbb{R}_{+}^{L_{t}} \times \mathbb{R}_{+}^{L_{t+1}}$ (or $\mathbb{R}_{+}^{L_{1}}$ for the generation 0) and satisfies $\bar{P}_{t}\left(\left(x^{i}\right)\right)+\left(\mathbb{R}_{+}^{L_{t}} \times \mathbb{R}_{+}^{L_{t+1}}\right) \subset \bar{P}_{t}\left(\left(x^{i}\right)\right)$ (or $\bar{P}_{t}\left(\left(x^{i}\right)\right)+\mathbb{R}_{+}^{L_{1}} \subset \bar{P}_{t}\left(\left(x^{i}\right)\right)$ for the generation 0$) . \bar{P}_{t}\left(\left(x^{i}\right)\right)$ is closed since $\bar{P}^{i}\left(x^{i}\right)$ is a nonempty closed subset of $\mathbb{R}_{+}^{L_{t}} \times \mathbb{R}_{+}^{L_{t+1}}$ for all $i \in \mathcal{I}_{t}$. Indeed, for all $t$ and for all $i \in \mathcal{I}_{t}$, since $\bar{P}^{i}\left(x^{i}\right)$ is closed, convex and comprehensive, its asymptotic cones $A \bar{P}^{i}\left(x^{i}\right)$, which coincides with its recession cones as defined in Rockafellar [22], is equal to $\mathbb{R}_{+}^{L_{t}} \times \mathbb{R}_{+}^{L_{t+1}}$. So, the asymptotic cones $\left(A \bar{P}^{i}\left(x^{i}\right)\right)_{i \in \mathcal{I}_{t}}$ are positively semi-independent, that is, for all $\left(\xi^{i}\right) \in \prod_{i \in \mathcal{I}_{t}} A \bar{P}^{i}\left(x^{i}\right)$ such that $\sum_{i \in \mathcal{I}_{t}} \xi^{i}=0$, then $\xi^{i}=0$ for all $i \in \mathcal{I}_{t}$. Consequently, from Debreu [12], the sum $\sum_{i \in \mathcal{I}_{t}} \bar{P}^{i}\left(x^{i}\right)$ is closed.

If $x=\left(x^{i}\right)$ is an interior allocation supported by the price $p \in \Delta$ and $x^{t}=\sum_{i \in \mathcal{I}_{t}} x^{i}$. Then one checks that for all $t \geq 0$, for all $i \in \mathcal{I}_{t}$,

$$
N_{\bar{P}_{t}\left(\left(x^{i}\right)\right)}\left(\bar{x}^{t}\right)=\left\{\lambda \Pi_{t} \mid \lambda \geq 0\right\}=\left\{\lambda \gamma^{i}\left(x^{i}\right) \mid \lambda \geq 0\right\}
$$

Definition 4 (a) For a given feasible allocation $x=\left(x^{i}\right)$, the sequence of commodity transfers $h=\left(h^{i}\right) \in \prod_{i \in \mathcal{I}_{0}} \mathbb{R}^{L_{1}} \times \prod_{t=1}^{\infty} \prod_{i \in \mathcal{I}_{t}}\left(\mathbb{R}^{L_{t}} \times \mathbb{R}^{L_{t+1}}\right)$ is feasible 
if $\left(x^{i}+h^{i}\right)$ is feasible, which means that $\left(x^{i}+h^{i}\right)$ belongs to $\prod_{t=0}^{\infty} \prod_{i \in \mathcal{I}_{t}} X^{i}$ and $\sum_{i \in \mathcal{I}_{t-1} \cup \mathcal{I}_{t}} h_{t}^{i}=0$.

(b) The sequence of commodity transfers $h=\left(h^{i}\right)$ is Pareto improving upon $x=\left(x^{i}\right)$ if $h$ is feasible and $x+h$ Pareto dominates $x$, that is for all $t \geq 1$ and all $i \in \mathcal{I}_{t}, x^{i}+h^{i} \in \bar{P}^{i}\left(x^{i}\right)$, with $x^{i}+h^{i} \in P^{i}\left(x^{i}\right)$ for at least one agent $i$.

(c) An aggregate transfer $\bar{h} \in \mathbb{R}^{L_{1}} \times \prod_{t=1}^{\infty} \mathbb{R}^{L_{t}} \times \mathbb{R}^{L_{t+1}}$ is feasible if $\bar{h}_{t}^{t-1}=-\bar{h}_{t}^{t}$ for all $t \geq 1$ and Pareto improving upon the allocation $x=\left(x^{i}\right)$ if:

i) for all $t, x^{t}=\sum_{i \in \mathcal{I}_{t}} x^{i}+\bar{h}^{t} \in \bar{P}_{t}\left(\left(x^{i}\right)\right)$

ii) there exists $\underline{t}$ such that $x^{\underline{t}}=\sum_{i \in \mathcal{I}_{\underline{t}}} x^{i}+\bar{h}^{\underline{t}} \in \operatorname{int}\left(\bar{P}_{\underline{t}}\left(\left(x^{i}\right)\right)\right)$

By the very definition of Pareto optimality, the allocation $x=\left(x^{i}\right)$ is Pareto optimal if and only if there exists no feasible Pareto improving transfer upon $x$. But, we also remark that the interior allocation $x=\left(x^{i}\right)$ is Pareto optimal if and only if there exists no feasible aggregate Pareto improving transfer upon $x$. So, in the next section, we will be able to work only on aggregate feasible transfers and not on feasible transfers, which will greatly simplify the notations and the formulas.

If there exists $\bar{h}$, an agregate Pareto improving transfer, then let $t \geq 0$, by definition of $\bar{P}_{t}\left(\left(x^{i}\right)\right)$, there exists $\left(\xi^{i}\right)_{i \in \mathcal{I}_{t}}$ in $\prod_{i \in \mathcal{I}_{t}} \bar{P}^{i}\left(x^{i}\right)$ such that $\sum_{i \in \mathcal{I}_{t}} x^{i}+$ $\bar{h}^{t}=\sum_{i \in \mathcal{I}_{t}} \xi^{i}$. By letting $h^{i}=\xi^{i}-x^{i}$, we easily check that $x^{i}+h^{i} \in \bar{P}^{i}\left(x^{i}\right)$ for all $i \in \mathcal{I}_{t}$.

Furthermore, for $\underline{t}, \sum_{i \in \mathcal{I}_{\underline{t}}} x^{i}+\bar{h}^{\underline{t}}-\alpha\left(\mathbf{1}_{L_{\underline{t}}}, \mathbf{1}_{L_{\underline{t}+1}}\right) \in \bar{P}^{\underline{t}}\left(\left(x^{i}\right)\right)$, for some $\lambda>0$ small enough. Then there exists $\left(\xi^{i}\right) \in \prod_{i \in \mathcal{I}_{\underline{t}}} \bar{P}^{i}\left(x^{i}\right)$ such that $\sum_{i \in \mathcal{I}_{\underline{\underline{t}}}} x^{i}+\bar{h}^{\underline{t}}-$ $\lambda\left(\mathbf{1}_{L_{\underline{t}}}, \mathbf{1}_{L_{\underline{t}+1}}\right)=\sum_{i \in \mathcal{I}_{\underline{t}}} \xi^{i}$. Let us consider the individual $i_{0} \in \mathcal{I}_{\underline{\underline{t}}}$, we can then write $\bar{h}^{\underline{t}}=\sum_{i \neq i^{0}}\left(\xi^{i}-x^{i}\right)+\left(\xi^{i_{0}}-x^{i}{ }_{0}\right)+\lambda\left(\mathbf{1}_{L_{\underline{t}}}, \mathbf{1}_{L_{\underline{t}+1}}\right)$. Take $h^{i}=\xi^{i}-x^{i}$ and $h^{i_{0}}=\xi^{i_{0}}-x^{i_{0}}+\lambda\left(\mathbf{1}_{L_{\underline{t}}}, \mathbf{1}_{L_{\underline{t}+1}}\right)$. We note that $x^{i_{0}}+h^{i_{0}}=\xi^{i_{0}}+\lambda\left(\mathbf{1}_{L_{\underline{t}}}, \mathbf{1}_{L_{\underline{t}+1}}\right) \in$ $P^{i_{0}}\left(\left(x^{i_{0}}\right)\right)$. So, $h$ is a Pareto improving transfer and $x$ is not Pareto optimal.

Conversely, if $x$ is not Pareto optimal, there exists $h$ a Pareto improving transfer. From the convexity of $P^{i}\left(x^{i}\right)$ (Assumption A(a)), (1/2)h is also a Pareto improving transfer. We now check that $\bar{h}$ defined by $\bar{h}^{t}=(1 / 2) \sum_{i \in \mathcal{I}_{t}} h^{i}$ is an aggregate Pareto improving transfer. $\bar{h}$ is obviously feasible since $h$ is feasible. For all $t$, since $(1 / 2) h$ is Pareto improving, $x^{t}+\bar{h}^{t} \in \bar{P}_{t}\left(\left(x^{i}\right)\right)$, with $x^{t}=\sum_{i \in \mathcal{I}_{t}} x^{i}$. Since $x^{i}$ is an interior allocation, $x^{i}+(1 / 2) h^{i}$ belongs to the interior of $X^{i}$ for all $i$. For the agent $i_{0}$ of generation $t_{0}$ such that $x^{i_{0}}+h^{i_{0}} \in P^{i_{0}}\left(x^{i_{0}}\right)$, since $P^{i_{0}}\left(x^{i_{0}}\right)$ is open (Assumption A(a)), one gets that there exists $\lambda>0$ such that $x^{i_{0}}+(1 / 2) h^{i_{0}}-\lambda\left(\mathbf{1}_{L_{\underline{t}}}, \mathbf{1}_{L_{\underline{t}+1}}\right) \in P^{i_{0}}\left(x^{i_{0}}\right)$. So, $\sum_{i \in \mathcal{I}_{t_{0}}} x^{i}+(1 / 2) h^{i}-\lambda\left(\mathbf{1}_{L_{\underline{t}}}, \mathbf{1}_{L_{\underline{t}+1}}\right) \in \sum_{i \in \mathcal{I}_{t_{0}}} \bar{P}^{i}\left(x^{i}\right)=\bar{P}^{t_{0}}\left(\left(x^{i}\right)\right)$, which implies that $\sum_{i \in \mathcal{I}_{t_{0}}} x^{i}+(1 / 2) h^{i} \in \operatorname{int}\left(\bar{P}_{t_{0}}\left(\left(x^{i}\right)\right)\right)$. 


\section{Characterization of Pareto-optimal allocations}

We now state the main result of the paper. It provides a condition on the supporting price of a weak Pareto optimal allocation, which is necessary and sufficient for the Pareto optimality of the given allocation.

Proposition 2 Let $x=\left(x^{i}\right) \in \prod_{i \in \mathcal{I}} X^{i}$ be a WPO allocation supported by the price sequence $p=\left(p_{1}, p_{2}, \ldots p_{t}, \ldots\right)$. We suppose that:

Assumption B: there exist $\bar{\chi}>0$ and $\underline{\chi}>0$ such that for all $t \geq 1, \bar{e}_{t} \leq \bar{\chi} \mathbf{1}_{L_{t}}$, $\underline{\chi}\left(\mathbf{1}_{L_{t}}, \mathbf{1}_{L_{t+1}}\right) \leq x^{i}$ for all $i \in \mathcal{I}_{t}$ and $\underline{\chi} \mathbf{1}_{L_{1}} \leq x^{i}$ for all $i \in \mathcal{I}_{0}$;

Assumption $C$ : there exists $\underline{r}>0$ such that for all $i \in \mathcal{I}, B\left(x^{i}+\underline{r} \gamma^{i}\left(x^{i}\right), \underline{r}\right) \subset$ $P^{i}\left(x^{i}\right)$;

Assumption $C^{\prime}$ : there exists $\bar{r}>0$ such that for all $i \in \mathcal{I}_{-0}$ (resp. $i \in \mathcal{I}_{0}$ ), for all $\xi^{i} \in \bar{P}^{i}\left(x^{i}\right)$, if $\xi^{i} \leq\left(e_{t}, e_{t+1}\right)$ (resp. $\left.\xi^{i} \leq e_{1}\right)$, then $\xi^{i} \in \bar{B}\left(x^{i}+\right.$ $\left.\bar{r} \gamma^{i}\left(x^{i}\right), \bar{r}\right)$;

Assumption $G$ : there exists $\bar{\nu} \geq \underline{\nu}>0$ such that for all $t \geq 1, i \in \mathcal{I}_{t}$,

$$
\underline{\nu} \leq \frac{\left\|\gamma_{t}^{i}\left(x^{i}\right)\right\|}{\left\|\gamma_{t+1}^{i}\left(x^{i}\right)\right\|} \leq \bar{\nu}
$$

Then, $x$ is Pareto optimal if and only if:

$$
\sum_{t \in \mathbb{N}^{*}} \frac{1}{\left\|p_{t}\right\|}=+\infty
$$

Since $x$ is supported by the price sequence $p=\left(p_{1}, p_{2}, \ldots p_{t}, \ldots\right)$, we recall that Lemma 1 implies that for all period $t$, for all $i \in \mathcal{I}_{t}, \frac{1}{\left\|\Pi_{t}\right\|} \Pi_{t}=$ $\frac{1}{\left\|\left(p_{t}, p_{t+1}\right)\right\|}\left(p_{t}, p_{t+1}\right)$ is equal to $\gamma^{i}\left(x^{i}\right)$. We denote by $\gamma^{t}$ the vector $\frac{1}{\left\|\Pi_{t}\right\|} \Pi_{t}$ and we let $x^{t}=\sum_{i \in \mathcal{I}_{t}} x^{i}$.

Remark 2 i) Assumptions C and C' mean that the boundaries of the preferred sets lie below small closed balls of radius $\underline{r}$ and above the comprehensive hull of bigger closed balls of radius $\bar{r}$. Assumptions $\mathrm{C}$ and C' means that the preferences are smooth and uniformly strictly convex. While Assumptions C and C' in [3] are stated in terms of curvature of the utility functions, we have chosen a more geometric approach because the preferences are not representable by a utility function.

For each individual $i$, let us consider the closed set $F^{i}$ defined as the complementery of $P^{i}\left(x^{i}\right), F^{i}:=\complement P^{i}\left(x^{i}\right)$. Then Assumption $\mathrm{C}$ means that $F^{i}$ is prox-regular at $x^{i}$. Indeed, let $r>0$ and $\beta>0$. The set $F$ is called $(r, \beta)$ prox-regular at $x \in F$, if for any $y \in F \cap B(x, \beta)$ and any $v \in N^{P}(F, y)$ with 
$\|v\| \leq 1, y \in \operatorname{Proj}_{F}(y+r v)$ where $N^{P}(F, y)$ is the proximal normal cone to $F$ at $y$, that is:

$$
N^{P}(F, y)=\left\{v \mid \exists \rho>0, y \in \operatorname{Proj}_{F}(y+\rho v)\right\}
$$

In our framework, under Assumption A,

$$
N^{P}(F, y)=\{\mu \gamma(y) \mid \mu \geq 0\}
$$

The notion of prox-regularity was introduced by Poliquin and Rockafellar, as a new important regularity in variational analysis, see [20], and extensively studied by Colombo and Thibault in [11].

ii) Note that Assumption B implies that the number of individuals is uniformly bounded above at each generation. Indeed, if $I_{t}$ is the number of individual of the generation $t$, and $h$ is a commodity at period $t$, then $I_{t} \chi \leq \sum_{i \in \mathcal{I}_{t}} x_{h}^{i} \leq$ $e_{t h} \leq \bar{\chi}$. We denote by $\bar{I}$, an upper bound of the number of individual at each generation.

iii) Assumptions C and C' still hold when we aggregate the finitely many consumers at each period by considering the set $\bar{P}^{t}\left(\left(x^{i}\right)\right)$. Assumption $\mathrm{C}$ implies $B\left(x^{t}+I_{t} \underline{r} \gamma^{t}, I_{t} \underline{r}\right) \subset \sum_{i \in \mathcal{I}_{t}} P^{i}\left(x^{i}\right)$, thus, whatever is the number of consumers of generation $t, B\left(x^{t}+\underline{r} \gamma^{t}, \underline{r}\right) \subset \operatorname{int} \bar{P}^{t}\left(\left(x^{i}\right)\right)$.

Let $\xi^{t} \in \bar{P}^{t}\left(\left(x^{i}\right)\right)$ such that $\xi^{t} \leq\left(e_{t}, e_{t+1}\right)$. Then Assumption C' implies that $\xi^{t}$ belongs to $\sum_{i \in \mathcal{I}_{t}} \bar{B}\left(x^{i}+\bar{r} \gamma^{i}\left(x^{i}\right), \bar{r}\right)$, which is equal to $\bar{B}\left(x^{t}+I_{t} \bar{r} \gamma^{i}\left(x^{i}\right), I_{t} \bar{r}\right)$. So uniformly in $t, \xi^{t}$ belongs to $\bar{B}\left(x^{i}+\bar{I} \bar{r} \gamma^{i}\left(x^{i}\right), \bar{I} \bar{r}\right)$.

For the coherence of the notations, we let $\rho=\underline{r}$ and $\bar{\rho}=\bar{I} \bar{r}$.

iv) We remark that Assumption G is slightly weaker than Property G in Balasko and Shell in [3]. Indeed, Property G assumes that the ratio $\frac{p_{s \ell}}{\left\|\Pi_{t}\right\|}$ is uniformly bounded above and away from 0 for all period $t$, for $s=t, t+1$ and for all commodities $\ell$ at date $t$ or $t+1$. Recalling that $\Pi_{t}$ is positively collinear to $\gamma^{t}$, this clearly implies that the ratio $\frac{\left\|\gamma_{t}^{t}\right\|}{\left\|\gamma_{t+1}^{t}\right\|}$ is uniformly bounded above and away from 0 for all period $t$.

Remark 3 If $\bar{h}$ is a feasible aggregate Pareto improving transfer upon $\left(x^{i}\right)$, then by Definition $4, x^{t}+\bar{h}^{t} \in \bar{P}^{t}\left(\left(x^{i}\right)\right)$, so thanks to Assumption C', $\Pi_{t} \cdot \bar{h}^{t}>0$, if $\bar{h}^{t} \neq 0$. Using this fact and the feasibility condition $\bar{h}_{t}^{t-1}=-\bar{h}_{t}^{t}$, we easily prove by induction that $\bar{h}^{t} \neq 0$ implies $\bar{h}^{t+1} \neq 0$. Since at least one generation $t_{0}$ is strictly better off after the transfer, $\bar{h}^{t_{0}} \neq 0$. So, there exist $\underline{t} \geq 0$ such that $\bar{h}^{t}=0$ for $t<\underline{t}$ and $\bar{h}^{t} \neq 0$ for $t \geq \underline{t}$.

\subsection{Proof of Proposition 2}

Since the allocation $x=\left(x^{i}\right)$ is Pareto optimal if and only if there exists no feasible aggregate Pareto improving transfer upon $x$, the proof will be established 
by constructing and characterizing a sequence of aggregate Pareto improving transfers.

Let $\bar{h}$ be a feasible aggregate transfer. Set $\eta^{t}:=\Pi_{t} \cdot \bar{h}^{t}$, the net present value of the aggregate transfer $\bar{h}$ at each date $t$.

When $\eta^{t} \neq 0$, let us define $\alpha^{t}$ by:

$$
\alpha^{t}:=\frac{\left\|\bar{h}^{t}\right\|^{2}\left\|\Pi_{t}\right\|}{\Pi_{t} \cdot \bar{h}^{t}}=\frac{\left\|\bar{h}^{t}\right\|^{2}\left\|\Pi_{t}\right\|}{\eta^{t}}
$$

Remark 4 i) Note that $\left\|\bar{h}^{t}\right\|^{2}=\frac{\alpha^{t} \eta^{t}}{\left\|\Pi_{t}\right\|} \leq \frac{\alpha^{t}\left\|\Pi_{t}\right\|\left\|\bar{h}^{t}\right\|}{\left\|\Pi_{t}\right\|}$, thus $\left\|\bar{h}^{t}\right\| \leq \alpha^{t}$. Thus, if $\alpha$ is bounded then $\bar{h}$ is also bounded.

ii) $\frac{\alpha^{t}}{2}$ actually represents the radius of the sphere $S\left(x^{t}+\frac{\alpha^{t}}{2} \gamma^{t}, \frac{\alpha^{t}}{2}\right)$ which is tangent to $\bar{P}^{t}\left(\left(x^{i}\right)\right)$ at $x^{t}$, and contains $x^{t}+\bar{h}^{t}$. Indeed, we easily check that:

$$
\left\|x^{t}+\bar{h}^{t}-\left(x^{t}+\frac{\alpha^{t}}{2} \gamma^{t}\right)\right\|=\frac{\alpha^{t}}{2}
$$

We prepare the proof by three lemmas and then we prove the necessary and the sufficient condition in two additional lemmas.

Lemma 3 Let $\bar{h}$ be a feasible aggregate Pareto improving transfer upon $x=$ $\left(x^{i}\right)$. Under Assumption $C^{\prime}$, from Remark 3, there exists $\underline{t} \geq 0$ such that $h^{t}=0$ for $t<\underline{t}$ and $h^{t} \neq 0$ for $t \geq \underline{t}$. Then the sequence $\alpha^{t}$ is bounded from above for all $t \geq \underline{t}$.

Proof. Since $\bar{h}$ is a feasible aggregate Pareto improving transfer, from Remark $3, \bar{h} \neq 0$ and $\eta^{t} \neq 0$ for all $t \geq \underline{t}$. Furthermore, $0 \leq \xi^{t}=x^{t}+\bar{h}^{t} \leq\left(e_{t}, e_{t+1}\right)$. So, from Assumption $C^{\prime}$ and Remark 2, for all $t \geq \underline{t}, \xi^{t} \in \bar{B}\left(x^{t}+\bar{\rho} \gamma^{t}, \bar{\rho}\right)$. Hence, from Remark 4 (ii), the sphere $S\left(x^{t}+\frac{\alpha^{t}}{2} \gamma^{t}, \frac{\alpha^{t}}{2}\right)$, which is tangent to $\bar{P}^{t}\left(\left(x^{i}\right)\right)$ at $x^{t}$, and contains $\xi^{t}$ is included in $\bar{B}\left(x^{t}+\bar{\rho} \gamma^{t}, \bar{\rho}\right)$. So $\frac{\alpha^{t}}{2} \leq \bar{\rho}$, which shows that $\alpha^{t}$ is bounded from above.

Lemma 4 Let $\bar{h}$ be a feasible aggregate Pareto improving transfer upon $x=$ $\left(x^{i}\right)$. Under Assumption $C^{\prime}$, from Remark 3, there exists $\underline{t} \geq 0$ such that $h^{t}=0$ for $t<\underline{t}$ and $h^{t} \neq 0$ for $t \geq \underline{t}$. Then, for all $t \geq \underline{t}$,

$$
\alpha^{t}=\frac{\left\|\Pi_{t}\right\|}{\eta^{t}}\left\|\bar{h}^{t}\right\|^{2} \geq \frac{\left\|\Pi_{t}\right\|}{\eta^{t}}\left[\frac{1}{\left\|p_{t}\right\|^{2}}\left(\eta^{0}+\ldots+\eta^{t-1}\right)^{2}+\frac{1}{\left\|p_{t+1}\right\|^{2}}\left(\eta^{0}+\ldots+\eta^{t}\right)^{2}\right]
$$

Proof. Indeed, the construction of $\eta$ and the feasibility of the transfer $h$ allow us to write:

$$
\begin{aligned}
& \eta^{0}=p_{1} \cdot \bar{h}^{0}=p_{1} \cdot \bar{h}_{1}^{0}=-p_{1} \cdot \bar{h}_{1}^{1} \\
& \eta^{1}=\Pi_{1} \cdot \bar{h}^{1}=p_{1} \cdot \bar{h}_{1}^{1}+p_{2} \cdot \bar{h}_{2}^{1}=-p_{1} \cdot \bar{h}_{1}^{0}+p_{2} \cdot \bar{h}_{2}^{1} \\
& \ldots \\
& \eta^{t}=\Pi_{t} \cdot \bar{h}^{t}=p_{t} \cdot \bar{h}_{t}^{t}+p_{t+1} \cdot \bar{h}_{t+1}^{t}=-p_{t} \cdot \bar{h}_{t}^{t-1}+p_{t+1} \cdot \bar{h}_{t+1}^{t}
\end{aligned}
$$


By summing up, we obtain that: $\eta^{0}+\eta^{1}+\ldots+\eta^{t}=p_{t+1} \cdot \bar{h}_{t+1}^{t}$. By definition, for $t \geq \underline{t}$,

$$
\alpha^{t}=\frac{\left\|\Pi_{t}\right\|}{\eta^{t}}\left(\left\|\bar{h}_{t}^{t}\right\|^{2}+\left\|\bar{h}_{t+1}^{t}\right\|^{2}\right)
$$

By Schwarz inequality, $\left(p_{t} \cdot \bar{h}_{t}^{t}\right)^{2} \leq\left\|p_{t}\right\|^{2}\left\|\bar{h}_{t}^{t}\right\|^{2}$, that is: $\left(\frac{p_{t} \cdot \bar{h}_{t}^{t}}{\left\|p_{t}\right\|}\right)^{2} \leq\left\|\bar{h}_{t}^{t}\right\|^{2}$, we then obtain that:

$$
\alpha^{t} \geq \frac{\left\|\Pi_{t}\right\|}{\eta^{t}}\left[\frac{\left(p_{t} \cdot \bar{h}_{t}^{t}\right)^{2}}{\left\|p_{t}\right\|^{2}}+\frac{\left(p_{t+1} \cdot \bar{h}_{t+1}^{t}\right)^{2}}{\left\|p_{t+1}\right\|^{2}}\right]
$$

Hence,

$$
\alpha^{t} \geq \frac{\left\|\Pi_{t}\right\|}{\eta^{t}}\left[\frac{1}{\left\|p_{t}\right\|^{2}}\left(\eta^{0}+\ldots+\eta^{t-1}\right)^{2}+\frac{1}{\left\|p_{t+1}\right\|^{2}}\left(\eta^{0}+\ldots+\eta^{t}\right)^{2}\right]
$$

Lemma 5 Let $\eta$ be a positive sequence in $\mathbb{R}$. Let us define an aggregate transfer $\bar{h}$ in $\mathbb{R}^{L_{1}} \times \prod_{t=1}^{\infty}\left(\mathbb{R}^{L_{t}} \times \mathbb{R}^{L_{t+1}}\right)$ and the associated $\alpha$ respectively by:

$$
\begin{gathered}
\bar{h}_{t+1}^{t}=\left(\eta^{0}+\eta^{1}+\ldots+\eta^{t}\right) \frac{p_{t+1}}{\left\|p_{t+1}\right\|^{2}} \text { and } \bar{h}_{t}^{t}=-\bar{h}_{t}^{t-1} \\
\alpha^{t}=\frac{\left\|\Pi_{t}\right\|}{\eta^{t}}\left\|\bar{h}^{t}\right\|^{2}=\frac{\left\|\Pi_{t}\right\|}{\eta^{t}}\left[\frac{1}{\left\|p_{t}\right\|^{2}}\left(\eta^{0}+\ldots+\eta^{t-1}\right)^{2}+\frac{1}{\left\|p_{t+1}\right\|^{2}}\left(\eta^{0}+\ldots+\eta^{t}\right)^{2}\right]
\end{gathered}
$$

Under Assumptions $B$ and $C$, if $\alpha$ is bounded then there exists $\mu>0$ such that $\mu \bar{h}$ is a feasible Pareto improving aggregate transfer.

Remark 5 In Lemma $5, \bar{h}$ is computed in such a way that $\Pi_{t} \cdot \bar{h}^{t}=\eta^{t}$ and the associated $\alpha^{t}$ is the smallest possible one.

Proof. Let $\mu>0$ be taken small enough so that $\mu \alpha^{t}<2 \rho$, for all $t$, which is feasible since $\alpha=\left(\alpha^{t}\right)$ is bounded.

From the formula above and Remark 4 (ii), since $\frac{\mu \alpha^{t}}{2} \leq \underline{\rho}, x^{t}+\mu \bar{h}^{t}$ belongs to the sphere $S\left(x^{t}+\frac{\mu \alpha^{t}}{2} \gamma^{t}, \frac{\mu \alpha^{t}}{2}\right) \subset B\left(x^{t}+\underline{\rho} \gamma^{t}, \underline{\rho}\right) \cup\left\{x^{t}\right\}$. Since $\bar{h}^{t} \neq 0, x^{t}+\mu \bar{h}^{t} \in$ $B\left(x^{t}+\underline{\rho} \gamma^{t}, \underline{\rho}\right) \backslash\left\{x^{t}\right\}$. From Assumption $\mathrm{C}$ and Remark 2 (iii), $x^{t}+\mu \bar{h}^{t} \in$ $\operatorname{int} \bar{P}^{t}\left(\left(x^{i}\right)\right)$, that is $\mu \bar{h}$ is a feasible aggregate Pareto improving transfer upon the allocation $\left(x^{i}\right)$.

Lemma 6 Given the positive price sequence $p$, if $x$ is not $P O$, then under Assumption $C^{\prime}, \sum_{t \in \mathbb{N}^{*}} \frac{1}{\left\|p_{t}\right\|}<+\infty$.

Proof. Since $x$ is not PO, there exists a Pareto improving aggregate transfer $\bar{h}$ such that $\bar{h}^{t}=0$ for $t<\underline{t}$ and $\bar{h}^{t} \neq 0$ for $t \geq \underline{t}$ for some $\underline{t} \geq 0$. From Lemma 3, $\alpha^{t}$ is bounded from above by $2 \bar{\rho}$ for $t \geq \underline{t}$, thus, from Lemma 4 , for all $t \geq \underline{t}$, 


$$
2 \bar{\rho} \geq \alpha^{t} \geq \frac{\left\|\Pi_{t}\right\|}{\eta^{t}\left\|p_{t+1}\right\|^{2}}\left[\eta^{0}+\ldots+\eta^{t}\right]^{2} \geq \frac{1}{\eta^{t}\left\|p_{t+1}\right\|}\left[\eta^{0}+\ldots+\eta^{t}\right]^{2}
$$

since $\frac{\left\|\Pi_{t}\right\|}{\left\|p_{t+1}\right\|}=\sqrt{1+\frac{\left\|p_{t}\right\|^{2}}{\left\|p_{t+1}\right\|^{2}}} \geq 1$. Thus:

$$
\frac{1}{\left\|p_{t+1}\right\|} \leq \frac{2 \bar{\rho} \eta^{t}}{\left[\eta^{0}+\ldots+\eta^{t}\right]^{2}}
$$

But, $\eta^{t}=0$ for $t<\underline{t}$, so,

$$
\frac{1}{\left\|p_{t+1}\right\|} \leq \frac{2 \bar{\rho} \eta^{t}}{\left[\eta^{\underline{t}}+\ldots+\eta^{t}\right]^{2}}
$$

Furthermore, for $t>\underline{t}$,

$$
\frac{\eta^{t}}{\left[\eta^{\underline{t}}+\ldots \eta^{t}\right]^{2}} \leq \frac{1}{\eta^{\underline{t}}+\ldots+\eta^{t-1}}-\frac{1}{\eta^{\underline{t}}+\ldots+\eta^{t}}
$$

This implies that:

$$
\sum_{t=\underline{t}+1}^{\infty} \frac{\eta^{t}}{\left[\eta^{\underline{t}}+\ldots+\eta^{t}\right]^{2}} \leq \frac{1}{\eta^{\underline{t}}}
$$

Hence

$$
\sum_{t=\underline{t}+2}^{\infty} \frac{1}{\left\|p_{t}\right\|}=\frac{2 \bar{\rho}}{\eta^{\underline{t}}}<+\infty
$$

hence $\sum_{t \in \mathbb{N}^{*}} \frac{1}{\left\|p_{t}\right\|}<+\infty$.

Remark 6 The following example shows that if preferences are flat at the given allocation, so not satisfying Assumption C', then the allocation could be not Pareto optimal even if $\sum_{t} \frac{1}{\left\|p_{t}\right\|}=+\infty$. Let us consider an OLG economy with one commodity per period, one consumer per generation, the allocation $(1,1)$ for all generation and the preferred set $\left\{\xi \in \mathbb{R}_{+}^{2} \mid t \xi_{t}+(t+1) \xi_{t+1}>2 t+1\right\}$. Then, one easily check that this allocation is not Pareto optimal because the allocation $(1 / 2,3 / 2)$ for each generation is Pareto dominating But, one also checks that the price $(1,2, \ldots, t, \ldots)$ supports this allocation and $\sum_{t} \frac{1}{\left\|p_{t}\right\|}=\sum_{t} \frac{1}{t}=+\infty$.

The last step of the proof of Proposition 2 is given by the following lemma.

Lemma 7 Under Assumptions $B, C$ and $G$, if the positive price sequence $p$ satisfies $\sum_{t} \frac{1}{\left\|p_{t}\right\|}<+\infty$, then $x$ is not $P O$.

Proof. Consider $\eta^{t}=\frac{1}{\left\|p_{t}\right\|}$ for all $t$, and denote by $\bar{\eta}^{t}:=\sum_{s=1}^{t} \eta^{s}$. Note that $\left(\bar{\eta}^{t}\right)$ is bounded. Let the corresponding agregate transfer $\bar{h}$ and the associated sequence $\alpha$ defined by the formula of Lemma 5. From this Lemma, it remains to show that $\alpha$ is bounded. 
From Lemma 5, we have: $\bar{h}^{t}=\left(\bar{\eta}^{t-1} \frac{p_{t}}{\left\|p_{t}\right\|^{2}}, \bar{\eta}^{t} \frac{p_{t+1}}{\left\|p_{t+1}\right\|^{2}}\right)$. Consequently, since $\bar{\eta}^{t-1} \leq \bar{\eta}^{t},\left\|\bar{h}^{t}\right\|^{2}=\left(\bar{\eta}^{t-1}\right)^{2} \frac{1}{\left\|p_{t}\right\|^{2}}+\left(\bar{\eta}^{t}\right)^{2} \frac{1}{\left\|p_{t+1}\right\|^{2}} \leq\left(\bar{\eta}^{t}\right)^{2}\left(\frac{1}{\left\|p_{t}\right\|^{2}}+\frac{1}{\left\|p_{t+1}\right\|^{2}}\right)$. Since $\eta^{t}=\frac{1}{\left\|p_{t}\right\|}$, the associated $\alpha$ defined in Lemma 5 satisfies:

$$
\begin{aligned}
\alpha^{t} & =\frac{\left\|\Pi_{t}\right\|}{\eta^{t}}\left\|\bar{h}^{t}\right\|^{2} \leq\left\|\Pi_{t}\right\|\left\|p_{t}\right\|\left(\bar{\eta}^{t}\right)^{2}\left(\frac{1}{\left\|p_{t}\right\|^{2}}+\frac{1}{\left\|p_{t+1}\right\|^{2}}\right) \\
& =\sqrt{\left\|p_{t}\right\|^{2}+\left\|p_{t+1}\right\|^{2}} \frac{2}{\left.\| \bar{\eta}^{t}\right)^{2}}\left(1+\frac{\left\|p_{t}\right\|^{2}}{\left\|p_{t}\right\|}\right) \\
& =\left(\bar{\eta}^{t}\right)^{2} \sqrt{1+\frac{\left\|p_{t+1}\right\|^{2}}{\left\|p_{t}\right\|^{2}}}\left(1+\frac{\left\|p_{t}\right\|^{2}}{\left\|p_{t+1}\right\|^{2}}\right)
\end{aligned}
$$

Assumption G implies that $\frac{\left\|p_{t}\right\|}{\left\|p_{t+1}\right\|}$ and $\frac{\left\|p_{t+1}\right\|}{\left\|p_{t}\right\|}$ are bounded. Since $\bar{\eta}^{t}$ is bounded, $\alpha$ is so.

Remark 7 The following example shows that if preferences exhibit a kink at the given allocation, so not satisfying Assumption $\mathrm{C}$, then the allocation could be Pareto optimal even if $\sum_{t} \frac{1}{\left\|p_{t}\right\|}<+\infty$. Let us consider an OLG economy with one commodity per period, one consumer per generation, the allocation $(1,1)$ for all generation and the same preferred set $\{(1,1)\}+\mathbb{R}_{++}^{2}$. Then, one easily checks that this allocation is Pareto optimal because to strictly improve the welfare of an agent, we need to reduce the allocation of the agent of the next generation when she is young so that her welfare strictly decreases. But, one also checks that the price $\left(1,2, \ldots, 2^{t}, \ldots\right)$ supports this allocation and $\sum_{t} \frac{1}{\left\|p_{t}\right\|}$ is finite.

\section{Conclusion}

Providing a simple set-theoretic and geometric version of the proof of Balasko and Shell [3] has allowed us to directly consider the multi-goods case, while in [3], the proof needs to first go through the one-commodity case. We have encompassed the case of non-complete and non-transitive preferences where basic assumptions are made on the preference sets and the associated normal cones. We provide a characterization of Pareto optimality that is established following a simple and geometric version of the proof of Balasko and Shell [3]. Moreover, we provide an explicit expression of improving transfers $h^{t}$ at each date $t$, that do not satisfy the criterion. Indeed, this is easily obtained thanks to the minimal value of the parameter $\alpha$ that is also used in [3].

A natural continuation to this work would be the extension to the case of OLG models with heterogeneous longevities within each generation and durable commodities. 


\section{References}

[1] Aumann R. J. (1962), Utility Theory without the Completeness Axiom, Econometrica, 30, 445-62.

[2] Balasko, Y., D. Cass and K. Shell (1980), Existence of Competitive Equilibrium in a General Overlapping Generations Model, Journal of Economic Theory, 23, 307-322.

[3] Balasko Y. and K. Shell (1980), The Overlapping Generations Model I: The Case of Pure Exchange without Money, Journal of Economic Theory, 23, 281-306.

[4] Bewley, T. (1986), Knightian decision theory: Part 1. Discussion paper N 807, Cowles Foundation, New Haven.

[5] Bewley, T. (1987), Knightian decision theory, Part II. Intertemporal problems, Cowles Foundation Discussion Paper 835.

[6] Bewley, T. (1988): Knightian decision theory and econometric inference, Cowles Foundation Discussion Paper 868.

[7] Bewley, T. (1989), Market innovation and entrepreneurship: A Knightian view, Cowles Foundation Discussion Paper 905.

[8] Borglin, A. (2004), Economic Dynamics and General Equilibrium: Time and Uncertainy, Springer-Verlag-Berlin, Heidelberg.

[9] Borglin, A. and H. Keiding (1986), Optimality in Infinite Horizon Economies, Lecture Notes in Economics and Mathematical Systems, Springer-Verlag Berlin Heidelberg.

[10] Burke J. L. (1987), Inactive Transfer Policies and Efficiency in General Overlapping Generations Economies, Journal of Mathematical Economics, 16, 201-222.

[11] Colombo G. and L. Thibault (2010), Prox-regular sets and applications, Handbook of Nonconvex Analysis, D.Y. Gao and D. Motreanu eds., International Press, 2010.

[12] Debreu G. (1959), Theory of value: An axiomatic analysis of economic equilibrium. Number 17 in Cowles Foundation Monographs. New Haven: Yale University Press.

[13] Florenzano M. (1981), L'équilibre économique général transitif et intransitif: Problèmes d'existence, Editions du CNRS. 
[14] Gale D. and A. Mas-Colell (1975), An equilibrium existence theorem for a general model without ordered preferences, Journal of Mathematical Economics, 2, 9-15.

[15] Gale D. and A. Mas-Colell (1979), Correction to an equilibrium existence theorem for a general model without ordered preferences, Journal of Mathematical Economics, 6, 297-298.

[16] Geanakoplos J. (2007), Overlapping Generations Models of General Equilibrium, Cowles Foundation Discussion Paper $n^{\circ} 1663$.

[17] Mandler, M. (2001a), A difficult choice in preference theory: rationality implies completeness or transitivity, but not both, Varieties of practical reasoning, ed. by E. Millgram. MIT Press.

[18] Mandler, M. (2001b), Incomplete preferences and rational intransitivity of choice, mimeo, University of London.

[19] Mas-Collel, A. (1974), An equilibrium existence theorem without complete or transitive preferences, Journal of Mathematical Economics, 1, 237 - 246.

[20] Poliquin R. A. and R. T. Rockafellar (1996), Prox-regular functions in variational analysis, Trans. Amer. Math. Soc. 348, 1805- 1838.

[21] Rakotonindrainy L. (2014), Existence of equilibrium in OLG economies with durable goods, Documents de travail du CES 2014.46, Université Paris 1 .

[22] Rockafellar R. T. and R. Wets (1998), Variational Analysis, SpringerVerlag, Berlin, Heidelberg, New York.

[23] Samuelson P. A. (1958), An Exact Consumption Loan Model of Interest, With or Without the Social Contrivance of Money, Journal of Political Economy, 66, 462-482. 